\title{
Insulin Resistance in Patients Undergoing Peritoneal Dialysis: Can We Improve It?
}

\author{
Editorial to: "The Effect of HM-CoA Reductase Inhibitor on Insulin Resistance \\ in Patients Undergoing Peritoneal Dialysis" by Fa Mee Doh et al.
}

\author{
Kelli King-Morris • T. Alp Ikizler
}

Published online: 17 November 2012

(C) Springer Science+Business Media New York 2012

Since 1972, there has been rapid growth in the end-stage renal disease (ESRD) program with the current growth rate between 5 and $7 \%$ per year. A recent report from the United States Renal Data System (USRDS) has indicated that there are over 410,000 maintenance dialysis patients, with Medicare expenditures of over 30 billion dollars to support the ESRD program in the year 2010 [1]. Approximately $30 \%$ of this total cost is due to hospitalization, which averages 14 days per patient year. Moreover, despite the many technical advances that have occurred in the delivery of dialysis therapy, the mortality rate of patients with ESRD remains high at over $20 \%$ per year.

Cardiovascular diseases (CVD) are the leading cause of morbidity and mortality in the United States and the presence of chronic kidney disease (CKD) is now recognized as an independent risk predictor for cardiovascular events. This is especially observed in advanced CKD such that CVD accounts for more than $40 \%$ of all deaths in the maintenance dialysis population [1]. In addition to conventional cardiovascular risk factors exemplified in the Framingham Study, uremia and dialysis-related metabolic disturbances such as chronic inflammation, increased oxidative stress burden and insulin resistance play a role to advance atherosclerosis leading to a life expectancy that is $25-30$ years shorter than matched controls $[2,3]$.

Amongst these metabolic disturbances, insulin resistance (IR), seems to play an important role, especially in peritoneal dialysis (PD) patients. Insulin resistance, the reciprocal of

K. King-Morris · T. A. Ikizler

Division of Nephrology, Department of Medicine,

Vanderbilt School of Medicine,

Nashville, TN, USA

T. A. Ikizler $(\square)$

Vanderbilt University School of Medicine,

116121 st Avenue South/MCN S-3312,

Nashville, TN 37232, USA

e-mail: alp.ikizler@vanderbilt.edu insulin sensitivity, describes a state of reduced biological effect for any given concentration of insulin in the plasma [4]. Insulin resistance plays a major pathophysiological role in glucose intolerance and type 2 diabetes mellitus (T2DM) and is tightly associated with major public health problems including obesity, hypertension, dyslipidemia, and atherosclerotic cardiovascular disease. Insulin resistance, measured by homeostatic model assessment (HOMA-IR), is reported to be common in CKD patients, including ones on PD and hemodialysis (HD) [5]. HOMA-IR is also shown to be an independent predictor of cardiovascular mortality in non-diabetic maintenance dialysis patients although the pathophysiological link has not been clearly delineated [6].

Alterations in insulin function associated with CKD were reported as early as 1951 and the effects of kidney disease on renal uptake and excretion of insulin was reported as early as 1970. DeFronzo and colleagues characterized uremic insulin resistance in patients with ESRD requiring dialysis using the euglycemic insulin clamp techniques demonstrating alterations in glucose metabolism in the face of hyperinsulinemia and diminished tissue sensitivity to insulin, which were partially correctable by hemodialysis [7]. Further studies demonstrated that altered insulin sensitivity was primarily due to a post-receptor defect altering primarily skeletal muscle rather than hepatic glucose uptake.

A unique aspect of PD that predisposes patients to insulin resistance is the inevitable glucose load from the dialysate [8]. By default, all non-diabetic PD patients are exposed glucose absorption from the peritoneum, with some at higher concentrations based on the dialysate required for ultrafiltration. A number of studies have shown that on average a CAPD patient absorbs in the range of $80-330$ grams of glucose from the dialysate [9]. Consequently, the prevalence of metabolic syndrome such as hyperglycemia, dyslipidemia and weight gain is increased in PD patients, $34 \%$ to $55 \%$ [10]. In PD patients, HOMA-IR is the most commonly used method to measure 
insulin resistance and there are very limited studies using gold standard insulin clamp study in this particular patient population. As an individual component of metabolic syndrome, insulin resistance is significantly higher in PD patients than in HD or pre-dialysis patients ( $47 \%$ vs $21 \%, 26 \%$ ) although other components of metabolic syndrome are not different between three groups [11]. Insulin resistance is also believed to result in a number of additional metabolic derangements, including but not limited to endothelial dysfunction, inflammation and increased oxidative stress. Indeed, numerous studies have shown increased concentrations of proinflammatory cytokines (Interleukin-6, Interleukin-1) and markers of oxidative stress (advanced oxidation protein products) in PD patients [12, 13]. Inhibition of the downstream signaling cascade by chronic inflammation leads to a reduction in response to ambient insulin levels. Insulin itself is anti-inflammatory, suppressing pro-inflammatory transcription factors such as NF-kappa beta and activating protein 1, all mediators of inflammation [14]. Derangement in the biologic effects of insulin begets more inflammation, which in turn begets decreased insulin sensitivity. Despite the well-defined information on the prevalence of "uremic insulin resistance", only a few investigations have centered on understanding the factors leading to insulin resistance in PD patients, its metabolic consequences and interventions to ameliorate IR in these patients.

In this issue of Cardiovascular Drugs and Therapy, Doh et al. [15] examined the effects of a specific HMG-CoA reductase inhibitor on insulin resistance in patients undergoing $\mathrm{PD}$ in a randomized clinical trial (RCT). They administered rosuvastatin $10 \mathrm{mg}$ per day or matching placebo for 6 months in 70 prevalent PD patients. Their results showed that rosuvastatin therapy failed to improve insulin resistance in PD patients despite a significant decline in LDL-cholesterol and hsCRP levels after statin treatment. More specifically, while there were numerical decreases in fasting insulin and glucose in the intervention arm, HOMA-IR, the primary measure of insulin resistance in this study, did not differ between groups. There were also no significant differences between groups in any of the adipokine concentrations, i.e. adiponectin, leptin and resistin measured during the study. They concluded that reducing inflammation by statin therapy is of limited help to fully attenuate insulin resistance in these patients.

The study by Doh and colleagues has multiple strengths. It is well designed and properly executed. The investigators provide a detailed analysis regarding rationale and the study outcomes and they provide a balanced interpretation of the study results. Overall, the investigators should be commended for carrying out an excellent study. There are also several limitations that warrant mention, which are also pointed out in the manuscript. For example, it is possible that the HMGCoA reductase inhibitor administered in this study may not the most appropriate one. If anything, recent epidemiological data indicate that use of rosuvastatin and several other statins (i.e. atorvastatin, simvastatin) are associated with increased risk of incident T2DM, whereas pravastatin seem to confer a benefit [16]. While the mechanisms leading to this intra-class difference are not fully elicited, it could be possible that use of a different statins might have provided a different result. As the authors point out, the dose administered in this study is similar to clinical practice. However, in order to elucidate the pleotropic effects of statins, higher doses may need to be administered. Finally, it is important to note that HOMA-IR has significant limitations as a marker of insulin sensitivity, despite the fact that it is the most commonly used marker in large epidemiological studies [17]. In smaller scale studies such as the one by Doh et al., it might be necessary to validate the results against the gold standard, i.e. hyper- or euglycemichyperinsulinemic clamp. While this might not a practical approach, studies comparing clamp studies to more practical methods are limited, especially in PD patients. Clamp studies provide a much more precise measure of changes in IR, centrally and peripherally. This has significant relevance to the study by Doh et al. since the apriori effect size on HOMAIR was estimated to $30 \%$, which is a quite significant change for an epidemiological marker and might have introduced a type II error.

The study by Doh et al. has clinical and research implications. At the very least, one can conclude that statin therapy has limited impact on IR observed in PD patients. The lack of biological effect could simply be related the unusual metabolic disturbances leading to IR in this patient population. This is a highly likely explanation since there was an obvious improvement in inflammatory profile as observed by statistically significant decrease in hsCRP levels in the absence of any changes in adipokine levels in response to rosuvastatin therapy. Regardless, future research for treatment of IR in PD patients should focus on alternative approaches. In that context, there is encouraging literature to support use of alternate osmotic agent such as icodextrin to decrease the amount of glucose exposure and absorption, which can amount to $1200 \mathrm{kcal}$ per day (300 g) on the usual dextrose containing solutions [18].

On the other hand, these results should not mean that use of statins has no role in PD patients. Dyslipidemia and its therapies have been a topic of great debate regarding its role in CKD progression, cardiovascular outcomes, and inflammation. Clinical trials such as AURORA and 4D failed to show benefit of statins in patients on chronic hemodialysis $[19,20]$; however, SHARP was powered by both predialysis and ESRD patients and was able to show reduction in myocardial infarctions, strokes, and requirement for revascularization, at least for CKD patients not on dialysis [21]. While the results did not reach significance for dialysis patients, PD patients have relatively more severe dyslipidemia compared to HD patients due to stimulation of hepatic lipoprotein synthesis due to continuous glucose absorption 
from the dialysate and represent a potential target for further intervention on this high risk population. Statin use is also of interest due to its pleotrophic effects and proven reduction in surrogate endpoints such as hsCRP, as shown in this study, as well as its beneficial effects of CVD prevention [22]. Chronic inflammation is observed in patients undergoing peritoneal dialysis and is strongly associated with cardiovascular disease and is a predictor of mortality. Inflammation is attributed to the mechanics of treatments including the presence of catheters and associated infections as well as the high-glucose containing dialysate and the activation of the complement cascade that ensues. Additionally, these patients have a tendency towards obesity, reported prevalence of $53 \%$, despite a continual loss of lean body mass, which results in increased inflammation. Central adipose tissue is metabolically active, and attracting macrophages and producing inflammatory mediators [23]. Immune players such as TNF-alpha activate and increase the expression of proteins that suppress the insulin-signaling pathway; ie insulin resistance. Continuous glucose absorption has been linked directly to ROS generation and also to increased central adiposity, which results in an inflamed state leading to the progression of atherosclerosis and damage to the vascular endothelium in PD patients.

In summary, the study by Doh et al. showed that 6 months of rosuvastatin therapy failed to improve insulin resistance in PD patients. This was observed despite a significant decline in LDL cholesterol and hsCRP levels after statin treatment. These results indicate that other strategies such as use of renin-angiotensin-aldosterone system blockade, insulin sensitizing agents and use of alternate osmotic agents, alone as well as in combination, should be examined to improve IR observed in PD patients. On the other hand, statins might have significant beneficial effects in PD patients, above and beyond any potential beneficial effects on insulin resistance, such as improvement in dyslipidemia and chronic inflammation. Dyslipidemia, chronic inflammation, and oxidative stress work in concert to orchestrate the development and progression of vascular endothelial damage, atherosclerosis, and cardiovascular events. Further well-designed studies, such as one performed Doh and colleagues, are necessary to improve at cardiovascular outcomes in PD patients.

\section{References}

1. Collins AJ, Foley RN, Chavers B, et al. 'United States Renal Data System 2011 Annual Data Report: atlas of chronic kidney disease $\&$ end-stage renal disease in the United States. Am J Kidney Dis. 2012;59(A7):e1-420.

2. Krane V, Wanner C. Statins, inflammation and kidney disease. Nat Rev Nephrol. 2011;7:385-97.
3. Himmelfarb J, Stenvinkel P, Ikizler TA, Hakim RM. The elephant in uremia: oxidant stress as a unifying concept of cardiovascular disease in uremia. Kidney Int. 2002;62:1524-38.

4. Hung AM, Ikizler TA. Factors determining insulin resistance in chronic hemodialysis patients. Contrib Nephrol. 2011;171:127-34.

5. Hung AM, Sundell MB, Egbert P, et al. A comparison of novel and commonly-used indices of insulin sensitivity in African American chronic hemodialysis patients. Clin J Am Soc Nephrol. 2011;6:767-74.

6. García-López E, Carrero JJ, Suliman ME, Lindholm B, Stenvinkel P. Risk factors for cardiovascular disease in patients undergoing peritoneal dialysis. Periton Dialysis Int. 2007;27:S205-9.

7. DeFronzo RA, Alvestrand A, Smith D, Hendler R, Hendler E, Wahren J. Insulin resistance in uremia. J Clin Invest. 1981; 67:563-8.

8. Fortes PC, de Moraes TP, Mendes JG, Stinghen AE, Ribeiro SC, Pecoits-Filho R. Insulin resistance and glucose homeostasis in peritoneal dialysis. Perit Dialysis Int. 2009;29:S145-8.

9. Grodstein GP, Blumenkrantz MJ, Kopple JD, Moran JK, Coburn JW. Glucose absorption during continuous ambulatory peritoneal dialysis. Kidney Int. 1981;19:564-7.

10. Chen HY, Kao TW, Huang JW, Chu TS, Wu KD. Correlation of metabolic syndrome with residual renal function, solute transport rate and peritoneal solute clearance in chronic peritoneal dialysis patients. Blood Purif. 2008;26:138-44.

11. Johnson DW, Armstrong K, Campbell SB, et al. Metabolic syndrome in severe chronic kidney disease: prevalence, predictors, prognostic significance and effects of risk factor modification. Nephrology (Carlton). 2007;12:391-8.

12. Cordeiro AC, Carrero JJ, Abensur H, Lindholm B, Stenvinkel P. Systemic and local inflammation in peritoneal dialysis: mechanisms, biomarkers and effects on outcome. Contrib Nephrol. 2009;163:132-9.

13. Nascimento MM, Suliman ME, Silva M, et al. Effect of oral Nacetylcysteine treatment on plasma inflammatory and oxidative stress markers in peritoneal dialysis patients: a placebo-controlled study. Perit Dialysis Int. 2010;30:336-42.

14. Hyun E, Ramachandran R, Hollenberg MD, Vergnolle N. Mechanisms behind the anti-inflammatory actions of insulin. Crit Rev Immunol. 2011;31:307-40.

15. Doh FM, Chang TI, Koo HM, et al. The effect of HMG-CoA reductase inhibitor on insulin resistance in patients undergoing peritoneal dialysis. Cardiovasc Drugs Ther 2012;26: this issue.

16. Koh KK, Sakuma I, Quon MJ. Differential metabolic effects of distinct statins. Atherosclerosis. 2011;215:1-8.

17. Wallace TM, Matthews DR. The assessment of insulin resistance in man. Diabet Med. 2002;19:527-34.

18. Babazono T, Nakamoto H, Kasai K, et al. Effects of icodextrin on glycemic and lipid profiles in diabetic patients undergoing peritoneal dialysis. Am J Nephrol. 2007;27:409-15.

19. Fellstrom BC, Jardine AG, Schmieder RE, et al. Rosuvastatin and cardiovascular events in patients undergoing hemodialysis. N Engl J Med. 2009;360:1395-407.

20. Wanner C, Krane V, Marz W, et al. Atorvastatin in patients with type 2 diabetes mellitus undergoing hemodialysis. N Engl J Med. 2005;353:238-48.

21. Baigent C, Landray MJ, Reith C, et al. The effects of lowering LDL cholesterol with simvastatin plus ezetimibe in patients with chronic kidney disease (Study of Heart and Renal Protection): a randomised placebo-controlled trial. Lancet. 2011;377:2181-92.

22. Ridker PM, Danielson E, Fonseca FAH, et al. Rosuvastatin to prevent vascular events in men and women with elevated C-reactive protein. N Engl J Med. 2008;359:2195-207.

23. Wellen KE, Hotamisligil GS. Inflammation, stress, and diabetes. J Clin Invest. 2005;115:1111-9. 